\title{
A Novel Two-Step Laser Ranging Technique for a Precision Test of the Theory of Gravity
}

\author{
Konstantin Penanen and Talso Chui ${ }^{\text {a * }}$

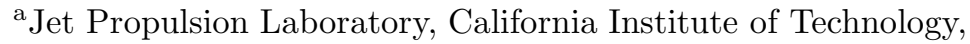 \\ 4800 Oak Grove Drive, Pasadena, CA 91109, USA
}

\begin{abstract}
All powered spacecraft experience residual systematic acceleration due to anisotropy of the thermal radiation pressure and fuel leakage. The residual acceleration limits the accuracy of any test of gravity that relies on the precise determination of the spacecraft trajectory. We describe a novel two-step laser ranging technique, which largely eliminates the effects of non-gravity acceleration sources and enables celestial mechanics checks with unprecedented precision. A passive proof mass is released from the mother spacecraft on a solar system exploration mission. Retro-reflectors attached to the proof mass allow its relative position to the spacecraft to be determined using optical ranging techniques. Meanwhile, the position of the spacecraft relative to the Earth is determined by ranging with a laser transponder. The vector sum of the two is the position, relative to the Earth, of the proof mass, the measurement of which is not affected by the residual accelerations of the mother spacecraft. We also describe the mission concept of the Dark Matter Explorers (DMX), which will demonstrate this technology and will use it to test the hypothesis that dark matter congregates around the sun. This hypothesis implies a small apparent deviation from the inverse square law of gravity, which can be detected by a sensitive experiment. We expect to achieve an acceleration resolution of $\sim 10^{-14} \mathrm{~m} / \mathrm{s}^{2}$. DMX will also be sensitive to acceleration towards the galactic center, which has a value of $\sim 10^{-10} \mathrm{~m} / \mathrm{s}^{2}$. Since dark matter dominates the galactic acceleration, DMX can also test whether dark matter obeys the equivalence principle to a level of 100 ppm by ranging to several proof masses of different composition from the mother spacecraft.
\end{abstract}

\section{Introduction}

Precise observations of motion of celestial bodies, along with corresponding theoretical models have formed the foundation of modern science. These include Kepler's insight into planetary motion based largely on Mars' ephemeris, Newton's subsequent formulation of the universal law of gravitation, and Einstein's explanation of Mercury perihelion advance. With increasing precision of modern measurement techniques it has become possible to determine the range to objects on the Moon to a centimeter level. Similar precision is routinely achieved in artificial satellite ranging. Science return of such measurements in-

\footnotetext{
*We would like to thank S.G. Turyshev and M.M. Nieto for helpful discussions. This work was carried out at the Jet Propulsion Laboratory, California Institute of Technology under a contract with the National Aeronautics and Space Administration. Contact: T. C. P. Chui (818)354-3104, Talso.C.Chui@jpl.nasa.gov
}

clude tests of strong equivalence principle (Lunar Laser Ranging (LLR), [12]) and general relativity tests (LAGEOS series [3]). While the precision of the range determination improves, the effects of non-gravitational influences on the studied objects becomes more significant. The observed anomalous $\sim 9 \times 10^{-10} \mathrm{~m} / \mathrm{s}^{2}$ acceleration of the Pioneer spacecraft towards the Sun 4 is an example of a potentially significant discovery obscured by the possible non-gravitation systematic effects. Pressure from $\sim 60$ Watts of unisotropically radiated heat from the spacecraft may account for the entire effect. Other typical non-gravitational influences include solar radiation and wind, propellant leakage, pressure due to directed radio communication, etc. Several techniques have been used to avoid or to mitigate the effects of non-gravitational systematics. Such effects would be small for objects that have large mass to surface area ratios. Laser ranging to 
retro-reflectors left by the Appollo and the Luna missions on the surface of the Moon, and the radio ranging to the Viking spacecraft on Mars make use of large masses of celestial bodies. Similar approach can be used by powered spacecraft only on or near a planet or an asteroid. LAGEOS Earth-orbiting retro-reflector satellites reduce the thermal radiation effects by not carrying power sources. Another ingenious work-around is the use of drag-free satellites (DISCOS, TRIAD, TIP, NOVA, LISA, STEP) where an inner object is shielded from the non-gravitational forces by an outer shell, which actively follows it. This technique requires substantial propellant expenditure. Moreover, in order to avoid gravitational interaction of the inner and outer bodies, the mass distribution of the spacecraft has to be extraordinarily well-defined.

In the context of gravitational science return from trajectory determination, the prospect of a nuclear-powered spacecraft with associated high-power communication subsystem presents a dilemma. With technology currently employed in lunar and satellite laser ranging, an optical transponder onboard would allow distance to such spacecraft to be measured with centimeter precision over hundreds of AU. At the same time, anisotropy in thermal radiation pressure due to the power source would alter the trajectory, making such a measurement meaningless. In this paper, we propose a novel two-step laser ranging technique which will circumvent the nongravitational systematic acceleration and will allow centimeter precision ephemeris determination on the 100-1000 AU scale.

\section{Tracking configuration}

Figure 1 illustrates the concept of the two-step ranging technique. Several proof masses, $1 \mathrm{~kg}$ each, of different compositions will be released from a mother spacecraft. Retro-reflectors will be attached to the surface of the proof masses. The mother spacecraft will follow the proof masses at a distance of $\sim 1 \mathrm{~km}$ away, so that the gravitational and electrostatic forces from the spacecraft would not affect the proof masses significantly. The distances to the proof masses will be deter-

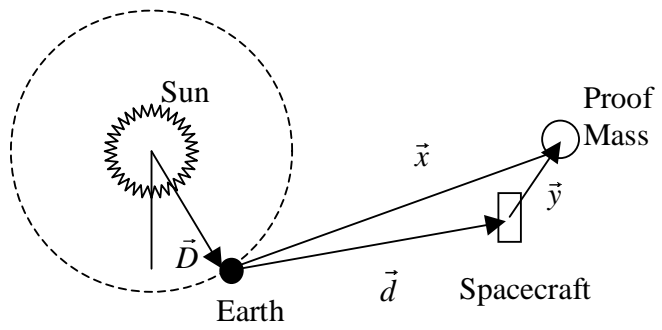

Figure 1. Illustration of the two-step ranging technique. Spacecraft experiences unpredictable parasitic acceleration, with resultant error in $\vec{d}$ and $\vec{y}$. Position of the proof mass relative to Earth, $\vec{x}$, is unaffected by spacecraft motion and can be determined from simultaneous measurement of $\vec{d}$ and $\vec{y}, \vec{x}=\vec{d}+\vec{y}$. Range from Earth to the proof mass, $\|\vec{x}\|$ can be determined to a few $\mathrm{cm}$.

mined by laser ranging from the mother spacecraft. The relative orientation of the proof masses will be determined by an optical telescope. The relative position of the proof masses can be determined with centimeter precision in a single measurement. The range to the spacecraft relative to the Earth will be inferred from the time it takes a laser beam to travel from the Earth to the spacecraft and back. The measurement is done using a laser transponder onboard the spacecraft, which sends back a reply beam to Earth after it detects a beam from Earth. Any time delay will be measured, and the information will be sent back to Earth together with the positions of the proof masses at a later time. The range of the proof mass relative to the Earth can then be determined. While the range to the Earth and and relative position of the proof masses are affected by residual motion of the mother spacecraft due to fuel leakage and uncontrolled thermal radiation pressure, the vector sum is not.

\section{Imaging and ranging}

The ranging laser on the spacecraft will be similar to the high-power YAG pulse lasers used in LLR [5]. The wavelength is $\sim 1 \mu m$, the beam 
divergence from a $25-\mathrm{cm}$ telescope is $\sim 10^{-5}$ radian, the pulse width is $\sim 100 \mathrm{ps}$, the energy for each pulse is $\sim 100 \mathrm{~mJ}$, a few pulses are fired in a train, and the pulse train is repeated at a rate of $10 \mathrm{~Hz}$. Thus the mean beam power for the laser is $P \approx 10 \mathrm{~W}$. With a 1 -meter telescope on Earth or on a near-Earth orbit and assuming quantum efficiency of 0.2 , we estimate $\sim 4$ photons per second detected by the receiver when the spacecraft is $1000 A U$ away. This rate is higher than that in a typical LLR experiment, where a reflected photon is detected every few seconds 2. If the error in range determination is limited by the $100 \mathrm{ps}$ pulse width, the range to the spacecraft can be determined to $\sim 3 \mathrm{~cm}$. Further improvement can be achieved by analyzing the pulse shape. Pulse coding and time shutter techniques similar to those of LLR can also be used. A similar on-board telescope in conjunction with a CCD will be used to image the proof masses. Estimated diffraction-limited lateral resolution at $1 \mathrm{~km}$ distance is $\sim 1 \mathrm{~cm}$. Ranging from the mother spacecraft to the proof masses will be achieved by sending a wide-beam pulse and analyzing time delay of the reflected signals.

\section{Error Sources}

We have evaluated known sources contributing to the error in range determination, and potential residual sources contributing to proof mass acceleration. Pulse width, clock drift, and atmospheric dispersion limit the range accuracy to a few centimeters in a 30-day measurement. Solar radiation uncertainty, solar wind, thermal radiation pressure, uncertainty in gravitational and electrostatic interaction with the mother spacecraft set a limit of $\sim 10^{-14} \mathrm{~m} / \mathrm{s}^{2}$. In combination, the systematic non-gravitational sources of error and errors of the range determination will allow acceleration measurements to $10^{-14} \mathrm{~m} / \mathrm{s}^{2}$ in a measurement lasting several tens of days.

\section{Science Goals}

We propose the described ranging scheme to be implemented on a deep space exploration mission as a guest experiment. Any deviation from the expected gravitational interaction of the proof masses with the solar system bodies will appear as anomalous correction to their trajectory. This experiment can be used to probe predictions of the Modified Newtonian dynamics and tensorscalar theories of gravitation on the Solar system scale, and to search for gravitational evidence of dark matter. While not sensitive to the average galactic dark matter density (which would require $\sim 10^{-16} \mathrm{~m} / \mathrm{s}^{2}$ acceleration resolution), the proposed scheme should detect any dark matter concentrated by Solar gravity by a factor of $\sim 100$ or more. Proof masses of different isotopic composition may also detect any equivalence principle violation in their attraction towards the galactic center to $100 \mathrm{ppm}$. The attraction is mostly due to dark matter. Kuiper belt and Oort cloud objects may produce additional short-term corrections to the acceleration. Quantifying interaction with such objects will allow better understanding of their abundance, mass and trajectory distributions.

\section{Conclusions}

At present there is a strong impetus from NASA to develop thermal nuclear energy and optical communication capability for deep space missions. With the proposed ranging scheme, these capabilities and the relevant infrastructure can be used to probe gravitational interactions on the Solar system scale with unprecedented precision. Our analysis indicates that centimeterlevel ranging resolution, and $10^{-14} \mathrm{~m} / \mathrm{s}^{2}$ acceleration resolution in a 30-day measurement can be achieved.

\section{REFERENCES}

1. J.G. Williams et al., Phys. Rev. Lett. 36, 551 (1976).

2. J.G. Williams, X.X. Newhall, and J.O. Dickey, Phys. Rev. D 53 (1996) 6739.

3. Ciufolini et al., Science 279 (1998) 2100.

4. J.D. Anderson et al., Phys. Rev. Lett. 81 (1998) 2858.

5. J.O. Dickey et al., Science 265 (1994) 482. 